\title{
Uma ferramenta colaborativa móvel para apoiar o processo de ensino-aprendizagem da LIBRAS e do Português para surdos
}

\author{
Elton Raniere da S. Moura, Elaine Harada T. de Oliveira \\ Instituto de Computação - Universidade Federal do Amazonas (UFAM) \\ Manaus - AM - Brasil \\ eltonranieremoura@gmail.com, elaine@icomp.ufam.edu.br
}

\begin{abstract}
This article presents a collaborative tool that aims to support teaching and learning LIBRAS and Portuguese for deaf students. A Systematic Literature Review (SLR) and a survey of the difficulties in the teachinglearning process in deaf education were held. A study of the application of a technique for collaborative learning in curricular activity was also conducted in order to observe the interactions between students and the role of communication during activities solving. As a result of the analysis of application activity, we proposed a collaborative tool based on a glossary model where this tool can be used by educators in group activities during class.
\end{abstract}

Resumo. O presente artigo apresenta uma ferramenta colaborativa que tem o objetivo de apoiar o processo de ensino-aprendizagem da LIBRAS e do português para alunos surdos. Foi realizada uma Revisão Sistemática de Literatura (RSL) e um levantamento sobre as dificuldades no processo de ensino-aprendizagem na educação de surdos. Também foi realizado um estudo da aplicação de uma técnica de aprendizagem colaborativa em atividade curricular, a fim de observar as interações entre os alunos e o papel da comunicação durante a resolução das atividades. Como resultado da análise da aplicação da atividade, foi proposta uma ferramenta colaborativa baseada num modelo de glossário, onde essa ferramenta poderá ser utilizada pelo educador em atividades em grupos durante as aulas.

\section{Introdução}

Segundo o resultado do Censo 2010 [IBGE], considerando a população residente no país, 39\% possuíam algum tipo de deficiência dentre as investigadas: visual, auditiva, motora, mental ou intelectual. Desse total $5,10 \%$ possuíam algum tipo de deficiência auditiva, sendo que a língua de sinais gestual é utilizada como principal meio de comunicação para essas pessoas. No entanto, quando os usuários da língua de sinais passam a se comunicar por meio da língua portuguesa (L2), encontram dificuldades na escrita, pois a estrutura sintática e morfológica da língua portuguesa se difere da LIBRAS (L1). No Brasil, o decreto $n^{0} 5.626$ [BRASIL, 2005] diz que as instituições de ensino devem oferecer atendimento especial aos alunos surdos em todos os processos que envolvem sua educação, como profissionais bilíngues, instrutores surdos e intérpretes de LIBRAS. O aprendizado da língua portuguesa torna-se necessidade em virtude das imposições da vida em sociedade, o que vai além dos fatores ligados aos processos de escolaridade. 
As Tecnologias de Informação e Comunicação (TIC) vem contribuindo com o processo de ensino-aprendizagem, provendo ferramentas que facilitam o acesso à informação e a interação entre pessoas, se adequando ao seu ritmo de aprendizagem. Com a internet móvel as pessoas passaram a trabalhar juntas na construção e organização de conhecimento, sem que estejam necessariamente no mesmo espaço físico. Nesse cenário é crescente o desenvolvimento e uso de sistemas colaborativos, que segundo Pimentel e Fuks [2011] tradicionalmente são sistemas computacionais desenvolvidos para dar suporte ao trabalho em grupo.

A proposta de uma ferramenta colaborativa móvel para apoiar o processo de ensino e aprendizagem da LIBRAS e do português surgiu de uma revisão sistemática de literatura sobre aprendizagem colaborativa, por meio de recursos computacionais para surdos. Foi realizada uma pesquisa com profissionais da educação especial para o levantamento de dificuldades durante o processo de ensino e aprendizagem, em seguida foi proposto desenvolver atividades na disciplina de língua portuguesa, através de Técnicas de Aprendizagem Colaborativa (Collaborative Learning Techniques - CoLTs). Segundo Barkley [2005, p. 288] quando se fala em aprendizagem colaborativa, significa trabalhar uns com os outros, ou seja, na prática são alunos trabalhando em pares ou grupos para atingir metas ou objetivos em comuns. A ferramenta colaborativa foi baseada no modelo de glossário, para que possa ser utilizada durante as aulas de língua portuguesa em atividades em grupo.

Este artigo está organizado conforme mostrado a seguir. Na seção seguinte, são apresentados os fundamentos teóricos, abordando a LIBRAS, o português como segunda língua e CoLTs. A seção 3 apresenta a Revisão Sistemática de Literatura (RSL), com o protocolo e condução da RSL. Na seção 4, é apresentada a proposta da ferramenta glossário e na seção 5 são apresentadas as considerações finais.

\section{Fundamentos Teóricos}

A proposta da ferramenta surgiu de uma Revisão Sistemática de Literatura e de um estudo sobre a educação especial para surdos. Através desse levantamento, pode-se propor uma metodologia de ensino-aprendizagem que possa ser utilizada durante as aulas, em atividades em grupo com uso da tecnologia. Após a pesquisa, foram identificadas técnicas de aprendizagem colaborativa e aplicadas em atividades na disciplina de língua portuguesa com alunos surdos.

\subsection{LIBRAS}

A LIBRAS surgiu sob influência da Língua de Sinais Francesa e da Língua Americana de Sinais chegando ao Brasil por volta de 1857, quando foi fundado o Instituto Nacional de Surdos-Mudos no Rio de Janeiro. Um estudo da ASL - (American Sign Language) foi realizado por Stokoe [1960, apud QUADROS e SCHMIEDT, 2006], com o objetivo de provar que a língua de sinais possui uma estrutura gramatical completa e não apenas uma língua baseada na comunicação das línguas orais de ouvintes. Diante disso a língua de sinais passou a ocupar a categoria de língua [QUADROS e SCHMIEDT, 2006].

Para construção dos sinais, é importante analisar a estrutura fonética da LIBRAS, que tem como principais características: a configuração das mãos, pontos de articulação, movimentos, orientação e expressão facial. A configuração de mãos indica o posicionamento das mãos para a realização do sinal; a pessoa usará a mão que 
"predomina" na realização das demais atividades, podendo ser esquerda ou direita. Ponto de articulação é o local onde incide a mão predominante, podendo estar em um espaço neutro ou tocar parte do corpo. O movimento é o terceiro parâmetro encontrado na língua de sinais que representa o deslocamento no espaço por parte das mãos, entretanto alguns sinais não possuem movimentos. A orientação é a direção que a palma da mão aponta na realização do sinal. Por fim, as expressões faciais se apresentam em dois tipos diferentes: expressões afetivas, que representam as características ligadas a sentimentos/emoções e as expressões gramaticais, que estão ligadas ao grau de adjetivos e ao grau de sentenças (afirmativas/negativas, interrogativas, exclamativas) [PIMENTA e QUADROS, 2006].

\subsection{Português como segunda língua (L2)}

A aquisição do português por alunos surdos é vista como a aquisição de uma segunda língua, da mesma forma como o inglês e espanhol, por exemplo. Para a aquisição dessa segunda língua por surdos, alguns aspectos fundamentais são apresentados [QUADROS e SCHMIEDT, 2006]:

- a existência de um alfabeto manual que faça referência visual com a língua portuguesa escrita;

- sistema de escrita alfabética que se difere da escrita da língua de sinais;

- os diferentes papéis sociais e acadêmicos que cada língua cumpre;

- a possibilidade de conversão da língua de sinais para o português;

- as diferentes categorias das línguas no processo educacional.

Os alunos dependem das habilidades de sua língua primária durante o processo de aprendizagem da língua secundária. Do ponto de vista cognitivo, o processo de aprendizagem da língua secundária é similar ao da língua primária, porém deve-se levar em conta a não existência do letramento na primeira língua. Nesse contexto é comum encontrar uma "Pedagogia surda" com sugestões para uma prática pedagógica adequada, pensada para quem vê e não para quem ouve, de forma a atingir todos os processos cognitivos que dependem do olhar [BAPTISTA et al., 2011].

\subsection{Técnicas de aprendizagem colaborativa}

A aprendizagem colaborativa segundo Matthews [1996, appud Barkley et al., 2005, p. 101], ocorre quando os educadores e alunos trabalham juntos na construção do conhecimento. Para os neurologistas e cientistas cognitivos, as pessoas "constroem" seu conhecimento ao longo da vida, conectando e organizando pedaços isolados de informação [Barkley et al., 2005].

As Técnicas de Aprendizagem Colaborativa - CoLTs - são técnicas eficazes para o desenvolvimento de atividades em grupo. Dessa forma, aprende-se através dos trabalhos em grupo ao invés de aprender trabalhando sozinho. Barkley et al. [2005, p. 216] apresenta trinta técnicas de aprendizagem colaborativa, dividida em cinco grupos, são elas: Techniques for Discussion; Techniques for Reciprocal Teaching; Techniques for Problem Solving; Techniques Using Graphic Information Organizers; Techniques Focusing on Writing. Essas técnicas são ferramentas simples e flexíveis, que podem ser facilmente adaptadas ao contexto das disciplinas e objetivos instrucionais. Um exemplo de atividade foi desenvolvido, a partir da técnica Group Grip - Using Graphic 
Information Organizers. Nela, os alunos trabalharam com identificação e organização de informações. Esta técnica é útil em cursos de níveis introdutórios, onde os alunos estão construindo um esquema básico, aprendendo um número grande de termos por exemplo. A organização e classificação de informações ajuda os alunos a esclarecer categorias conceituais e desenvolver habilidade de categorização [Barkley et al., 2005].

A atividade consistiu em pedir para que os alunos identificassem objetos dentro da sala de aula e em seguida inseri-los em um glossário apresentando o nome do item, uma frase curta contendo o nome do item e a configuração de mãos do item identificado. A educadora dividiu a turma em pequenos grupos com no máximo 3 alunos; cada grupo deveria identificar o maior número de objetos diferentes e anotá-los. Por fim, cada grupo deveria elaborar frases em português, com os nomes dos objetos identificados. Durante a realização da atividade, a comunicação entre os alunos e o professor foi feita em língua de sinais. Um aluno de cada grupo apresentou aos demais colegas da classe os objetos catalogados e durante a apresentação, os demais alunos puderam ajudar o colega orador, quando não sabia o sinal para o objeto. Durante a explicação a datilologia foi muito utilizada (soletração de uma palavra em português utilizando o alfabeto manual da língua de sinais). Na última fase da atividade, os alunos escreveram no quadro branco as frases desenvolvidas e os demais alunos puderam fazer suas contribuições e correções.

Após a realização desta atividade foi possível coletar informações que possam contribuir para a sua realização, utilizando a ferramenta glossário. A educadora da disciplina argumentou sobre a necessidade e importância da configuração de mãos para o surdo, uma vez que, com certa frequência, ele sabe o sinal referente à palavra, porém não sabe sua escrita. Com isso, foi proposta uma ferramenta glossário que o aluno possa inserir itens de acordo com a língua portuguesa ou de acordo com as configurações de mãos visíveis. Para tal, surgiu a necessidade de se verificar na literatura se algo semelhante já havia sido realizado.

\section{Revisão Sistemática de Literatura}

A RSL consiste em um levantamento rigoroso e metódico de pesquisas e estudos que procuram caracterizar um assunto ou uma tecnologia a partir da identificação, avaliação e interpretação dos resultados obtidos através de um processo pré-definido de coleta de dados [KITCHENHAM, 2004]. Segundo BIOLCHINI [2005], a RSL é um tipo de estudo secundário que tem por objetivo identificar tópicos ou questões de pesquisa ou fenômenos de interesse. Os estudos secundários exigem precisão e confiabilidade, para direcionar de forma eficiente a investigação científica [MAFRA, 2006].

Neste caso, a RSL coloca-se como uma estratégia adequada à realização de um levantamento do estado da arte sobre aprendizagem colaborativa em ambientes de ensino-aprendizagem para surdos.

\subsection{Protocolo da RSL}

Foi realizada uma Revisão Sistemática de Literatura (RSL) acerca do assunto e elaborado um Relatório Técnico. O relatório foi construído seguindo os tópicos, como apresentado a seguir.

1. No escopo da pesquisa foi estabelecido um modelo de pesquisa com base em: 
a. Critérios adotados para a escolha das Bibliotecas Digitais: As bases consultadas foram a IEEE, SCOPUS, SBIE e WIE.

b. Restrições: A pesquisa foi restrita à análise de publicações obtidas, exclusivamente, a partir das fontes selecionadas de acordo com dos critérios de inclusão e exclusão estabelecidos.

2. Idiomas: definição das línguas, sendo o Português para as bases nacionais e o Inglês para as bases internacionais.

3. Métodos de busca das publicações: descreveram-se as bases digitais onde foram realizadas as buscas.

a. Expressão de busca: apresentaram-se as strings usadas para as buscas nas bases de dados selecionadas, seguindo os critérios definidos para a População e Intervenção.

i. string 1: (("Lingua Brasileira de Sinais" OR "LIBRAS") AND ("educação" OR "aprendizagem" OR "aprendizagem colaborativa"))

ii. string 2: (("Brazilian language of signs" OR "LIBRAS") AND ("learning" OR "m-learning" OR "e-learning" OR "collaborative learning" OR "lms" OR "learning management system"))

4. Procedimentos de Seleção e Critérios: descreveram-se os procedimentos de seleção, através de uma estratégia de busca com o objetivo de identificar as publicações em potencial. Os critérios apresentam as regras para inclusão ou exclusão das publicações.

a. Critérios de Exclusão [ $1^{\circ}$ filtro]: foram excluídos os artigos cujos termos de busca não apareciam no título, palavras-chave, resumo ou abstract. As publicações que não citavam um ambiente de aprendizagem para ensino da LIBRAS também foram excluídas.

b. Critérios de Inclusão [ $1^{\mathrm{o}}$ filtro]: as publicações que apresentaram iniciativas relacionadas a ambientes de aprendizagem para ensino de LIBRAS foram incluídas.

5. Procedimentos para extração de dados: para extração dos dados das publicações selecionadas após o $1^{\mathrm{o}}$ filtro, os artigos foram armazenados e organizados conforme os itens a seguir: título, autor(es), palavras-chave, fonte da publicação, ano da publicação e abstract da publicação. Durante a leitura das publicações foram coletados dados derivados das características de interesse, que são: recursos utilizados, relatos de dificuldades, restrições e aplicabilidade.

6. Procedimentos para análise: foi realizada uma análise quantitativa, pela extração direta dos dados a partir do banco de dados com os registros dos achados. Em seguida, foi realizada uma análise qualitativa, a fim de estabelecer a relevância, as recomendações e as estratégias adotadas pelas implementações descritas nas publicações retornadas pela pesquisa.

A partir dessas publicações, foram realizadas etapas detalhadas de mapeamento definidas nos tópicos: planejamento, condução, análise e publicação dos resultados. 


\subsection{Condução da RSL}

No planejamento, foi elaborado um protocolo de estudo derivado do trabalho produzido por Souza [2008] e Kitchenham [2004]. Na etapa de condução, foram colhidas as publicações resultantes da busca nas bases digitais selecionadas. São elas: IEEE, SCOPUS, SBIE e WIE.

A pesquisa foi conduzida durante os meses de outubro de 2013 a janeiro de 2014. Com base nas publicações que correspondem ao grupo de controle, foram preparadas strings de busca a partir das palavras-chaves e dos termos relacionados. A busca resultou em 77 publicações, dos quais para a string 1 (Português) retornou em 40 publicações, a string 2 (Inglês) retornou em 37 publicações.

Foram realizadas buscas em bases nacionais modificando a string 1, reduzindo a busca com as palavras chaves: "LIBRAS" e "surdos". As bases consultadas, assim como os resultados das buscas foram: SBIE: 14 publicações e WIE: 8 publicações. Essa modificação na string 1 foi feita, pois não houve retorno de publicações com o uso da stringl definida no tópico 3.1 da sessão anterior.

Total de publicações

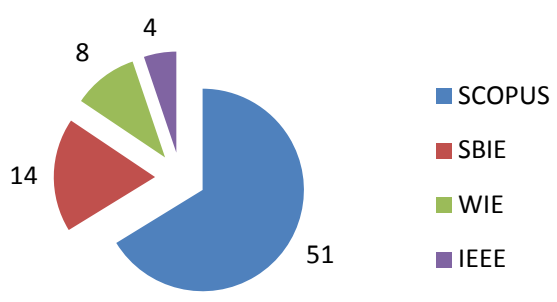

\section{Gráfico 1. Publicações retornadas a partir das expressões de busca.}

Das publicações retornadas, todos os títulos, abstracts e palavras-chave foram lidos. A análise para o $1^{\circ}$ filtro foi realizada segundo os critérios de inclusão e exclusão apresentados no protocolo de RSL. O Gráfico 2 a seguir, mostra a distribuição das publicações que passaram pelo $1^{\circ}$ filtro. As 26 publicações foram lidas novamente para extração de dados. O resultado deste processo retornou publicações que apresentavam aplicações com referências à aprendizagem da LIBRAS ou do Português como segunda língua.

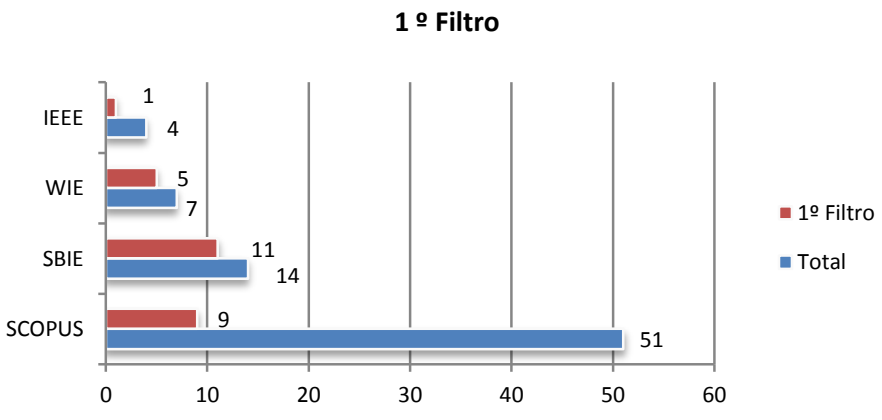

Gráfico 2. Publicações após o $1^{\circ}$ filtro. 


\subsection{Resultados}

Das 26 publicações selecionadas após o $1^{\circ}$ filtro, duas estavam relacionadas a Aprendizagem, LIBRAS e Colaboração. Dez publicações estavam relacionadas a Aprendizagem e Libras. As demais publicações apenas faziam referência a LIBRAS, mas sem relação com os demais critérios de busca. As aplicações citadas nas 12 publicações relacionadas à aprendizagem de LIBRAS são apresentadas na Tabela $1 \mathrm{a}$ seguir.

Tabela 1. Resultado das publicações relacionadas a Aprendizagem e Libras.

\begin{tabular}{|l|c|c|c|c|}
\hline \multicolumn{1}{|c|}{ Aplicação } & Aprendizagem & Libras & Colaboração & Base \\
\hline FALIBRAS & $\operatorname{Sim}$ & $\operatorname{Sim}$ & Não & SBIE \\
\hline SIGNHQ & $\operatorname{Sim}$ & $\operatorname{Sim}$ & Não & SBIE \\
\hline Human LIBRAS & $\operatorname{Sim}$ & $\operatorname{Sim}$ & Não & SBIE \\
\hline SignWebEDIT & $\operatorname{Sim}$ & $\operatorname{Sim}$ & $\operatorname{Sim}$ & SBIE \\
\hline Agente Pedagógico Clóvis & $\operatorname{Sim}$ & $\operatorname{Sim}$ & Não & SBIE \\
\hline SensorLibras & $\operatorname{Sim}$ & $\operatorname{Sim}$ & Não & SBIE \\
\hline SAEPS & $\operatorname{Sim}$ & $\operatorname{Sim}$ & Não & SBIE \\
\hline AVA CEAD & $\operatorname{Sim}$ & $\operatorname{Sim}$ & Sim & WIE \\
\hline InfoLIBRAS & $\operatorname{Sim}$ & $\operatorname{Sim}$ & Não & WIE \\
\hline ToonTalk & $\operatorname{Sim}$ & $\operatorname{Sim}$ & Não & WIE \\
\hline Agente Virtual Prof.' Libriane & $\operatorname{Sim}$ & $\operatorname{Sim}$ & Não & WIE \\
\hline Sistema Tutor Inteligente & $\operatorname{Sim}$ & $\operatorname{Sim}$ & Não & WIE \\
\hline
\end{tabular}

Ainda há poucos trabalhos envolvendo sistemas e ambientes colaborativos de aprendizagem para surdos. Do resultado obtido, somente as bases nacionais SBIE e WIE (Gráfico 3), apresentaram maior número de publicações selecionadas para um estudo aprofundado e extração de dados.

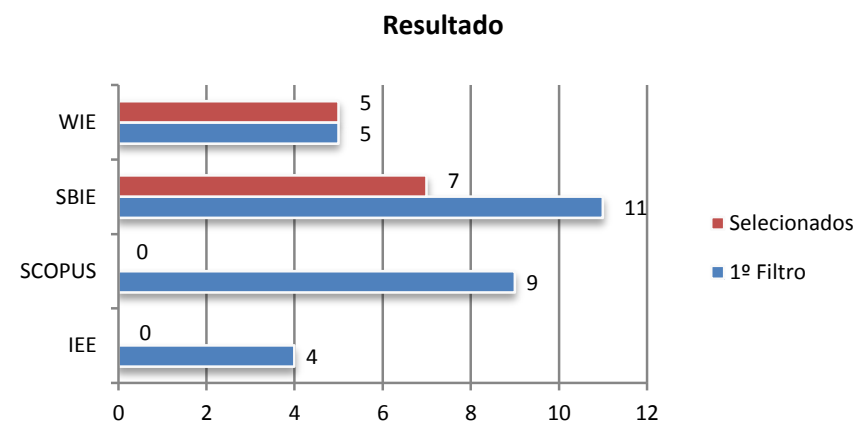

Gráfico 3. Publicações após o 1ำ filtro.

A partir do estudo das publicações que resultaram da RSL e da pesquisa com profissionais da educação especial, foram propostas novas metodologias, modeladas e aplicadas em atividades com a finalidade de promover atividades em grupo. Com esse estudo foi possível coletar mais informações sobre o processo de ensino $\mathrm{e}$ aprendizagem, que puderam contribuir para propor uma ferramenta que possa se adequar à metodologia empregada nas atividades em sala de aula.

O estudo apontou que ainda existem poucos trabalhos voltados ao ensino e aprendizagem do surdo, diante das inúmeras dificuldades e desafios encontrados no 
ambiente educacional. Para uma evolução positiva desse quadro atual, entende-se que é importante que haja estudos mais aprofundados e uma maior aproximação dos pesquisadores a esse ambiente. Conhecer e vivenciar um pouco desse "mundo particular" do surdo abre a mente para inúmeras possibilidades diante das grandes necessidades presentes.

\section{Proposta da ferramenta glossário}

O glossário colaborativo proposto parte da aplicação de técnicas de aprendizagem colaborativa em atividades da disciplina de língua portuguesa para alunos surdos. Com a realização da atividade citada na seção 3.1 deste trabalho, foram coletadas e analisadas informações decorrentes da aplicação de técnicas de aprendizagem colaborativa em uma atividade realizada para a disciplina. O modelo padrão de glossário com inserção, busca e visualização (ordem alfabética e categoria) será mantido, porém modificações foram propostas sugerindo à aplicação a navegação por configuração de mãos. Com essa modificação o aluno que sabe o sinal referente à palavra, mas não sua grafia ou datilologia terá maior facilidade para o preenchimento do glossário. As Figuras 1 e 2 a seguir representadas pelas letras A, B, C e D ilustram o protótipo da aplicação em desenvolvimento.

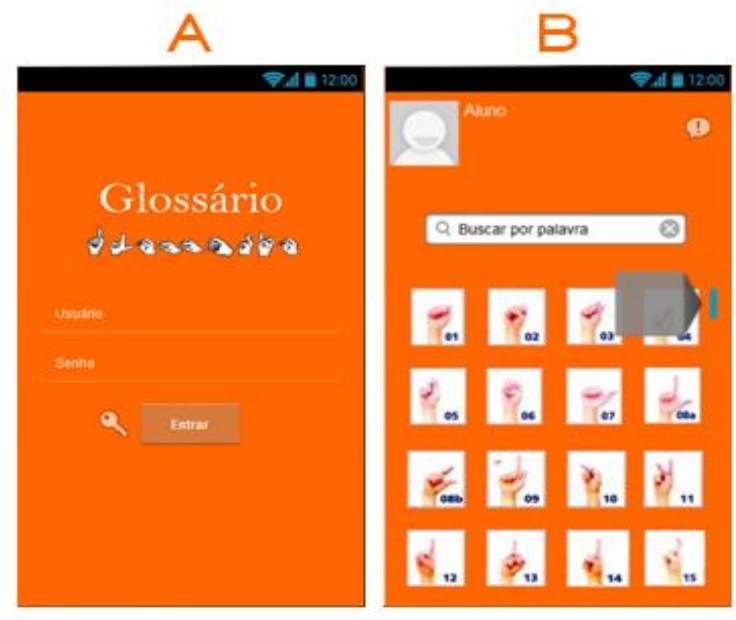

Figura 1. Tela A e B da aplicação glossário.
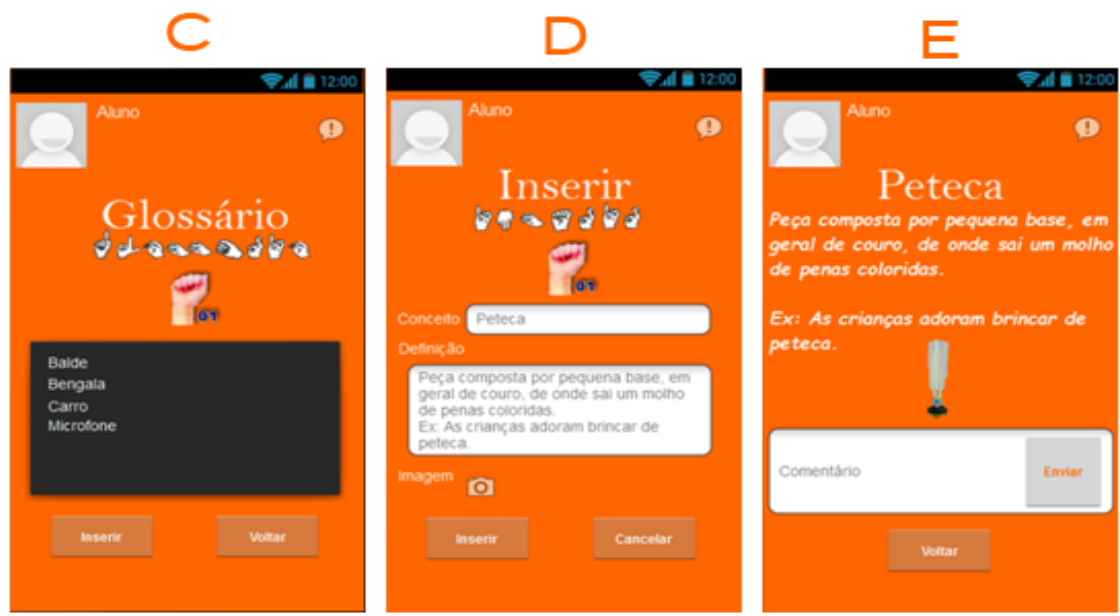

Figura 2. Tela C, D e E da aplicação glossário. 
Na Figura 1, a tela A apresenta a seção inicial do aplicativo, onde o aluno deverá inserir seus dados para entrada no sistema. A tela B apresenta a visão principal do glossário com as configurações de mãos disponíveis e a opção de busca por palavras em português. Na Figura 2, a tela $\mathrm{C}$ apresenta a visualização de uma configuração de mãos, listando os itens inseridos e opção de inserir um novo item. A tela D mostra os campos para inserção de um novo item com conceito, descrição e imagem (opcional). Por fim, a tela $\mathrm{E}$ mostra as informações de um item selecionado e também possibilita ao usuário inserir um comentário sobre o mesmo. A colaboração no aplicativo será dada seguindo a classificação 3C de sistemas colaborativos, que são: comunicação, coordenação e cooperação, como mostra a Tabela 2, a seguir. De maneira geral, a comunicação representa a troca de informações que pode ser dada por meio de mensagem de texto, a coordenação representa a administração de pessoas, ações e recursos, e a cooperação representa o comportamento conjunto no espaço compartilhado, que pode gerar produtos ou informações. O modelo $3 \mathrm{C}$ de colaboração, proposto por Teufel et al. [2000] e Borghoff \& Schlichter [2000] [apud PIMENTEL et al., 2006], é muito usado para classificar sistemas colaborativos.

Tabela 2. Serviços $3 \mathrm{C}$ do glossário.

\begin{tabular}{|c|c|c|}
\hline Comunicação & Cooperação & Coordenação \\
\hline Comentário & $\begin{array}{c}\text { Informações dos itens no } \\
\text { glossário. Informações de } \\
\text { comentários. }\end{array}$ & Avisos e Notificações \\
\hline
\end{tabular}

A aplicação será desenvolvida para sistema android, fazendo uso de linguagem Java, PHP e banco de dados MySQL. Todo sistema funcionará online para acesso e inserção de novos dados, pra isso será configurado um WebService para fazer o armazenamento e gerenciamento de informações.

A proposta da aplicação é que ela possa contribuir com a disciplina de língua portuguesa, através de atividades colaborativas definidas pelo professor e que se adaptem ao conteúdo programático da disciplina. Através do glossário os alunos poderão fazer a troca de informações e conteúdos, facilitando, assim, a construção de habilidades para a aquisição de conhecimento, compreensão, aplicação e criação. Além dos serviços 3C identificados na aplicação, o glossário permite a colaboração, no sentido de que o glossário como um todo é criado por todos.

\section{Considerações Finais}

Diante da RSL e dos relatos de profissionais da educação especial, verificou-se uma grande dificuldade no aprendizado do português como segunda língua para surdos e uma carência em aplicações computacionais voltadas a essas pessoas. Através da pesquisa realizada foram aplicadas técnicas de aprendizagem colaborativa em atividades na disciplina de língua portuguesa, de onde foi possível moldar uma ferramenta colaborativa para apoiar o processo de ensino-aprendizagem da LIBRAS e do português. Os testes em atividades educacionais com alunos apontaram diferentes características que posteriormente podem contribuir com futuros projetos computacionais na área de aprendizagem colaborativa.

A ferramenta colaborativa está em fase de desenvolvimento e posteriormente será realizado um estudo de caso aplicando a ferramenta em atividades com alunos surdos orientados por um professor da educação especial. 


\section{Referências}

BARKLEY, E.F.; CROSS, K.P.; MAJOR, C. H.. Collaborative Learning Techniques: A Handbook for College Faculty. (2005), San Fransisco, CA: Jossey-Bass.

BAPTISTA, José A.; SANTIAGO, Ana; ALMEIDA, Dina; ANTUNES, Paula; GASPAR, Regina. Programa De Português L2 Para Alunos Surdos - Ensino Básico e Secundário. Ministério da Educação. Direção-Geral de Inovação e Desenvolvimento Curricular - DGIDC. 15 de fevereiro de 2011.

BIOLCHINI, J.; MIAN, Paula Gomes; NATALI, Ana Candida Cruz; TRAVASSOS, Guilherme Horta. Systematic Review in Software Engineering. Technical Report, COPPE/UFRJ, 2005. Disponível em: <www.cin.ufpe.br/ in1037/leitura/systematic ReviewSE-COPPE.pdf>. Acesso em: 12 abr. 2010.

BRASIL. Decreto n. ${ }^{\circ} 5626$, de 22 de dezembro de 2005, Regulamenta a Lei $\mathrm{n}^{\circ} 10436$, de 24 de abril de 2002, que dispõe sobre a Língua Brasileira de Sinais - LIBRAS, e o art. 18 da Lei $\mathrm{n}^{0}$ 10098, de 19 de dezembro de 2000. Disponível em: $<$ http://www.planalto.gov.br/ccivil_03/_ato2004-2006/2005/decreto/d5626.htm. Acesso em Dezembro 5, 2013.

IBGE - Instituto Nacional de Geografia e Estatística. Cartilha do Censo 2010 - Pessoas com Deficiência. Secretaria Nacional de Promoção dos Direitos da Pessoa com Deficiência. Disponível em: < http://www.pessoacomdeficiencia.gov.br/app/sites/def ault/files/publicacoes/cartilha-censo-2010-pessoas-com-deficienciareduzido.pdf.

Acesso em 08 de outubro de 2014.

KITCHENHAM, B. Procedures for Performing Systematic Reviews. Joint Technical Report TR/SE-0401. Software Engineering Group, Department of Computer Science, Keele University, Australia, 2004. Disponível em: $<$ http://www.idi.ntnu.no/emner /empse/papers/kitchenham_2004.pdf>. Acesso em: 15 mar. 2010.

MAFRA, S. N.; TRAVASSOS, G. H. Estudos Primários e Secundários Apoiando a Busca por Evidência em Engenharia de Software. Technical Report, PESC COPPP/UFRJ, 2006. Disponível em: <www.cos.ufrj.br/uploadfiles/11491031 20.pdf $>$. Acesso em: 12 mar. 2010.

PIMENTA, Nelson; QUADROS, Ronice Muller de. Curso de Libras 1. Rio de Janeiro: LSB Vídeo, 2006.

PIMENTEL, Mariano; FUKS, Hugo. Modelos de Sociedades. Sistemas Colaborativos. Rio de Janeiro: Elsevier Editora Ltda - Campus, 2011.

PIMENTEL, Mariano; GEROSA, Marco Aurélio; FILIPPO, Denise; RAPOSO, Alberto; FUKS, Hugo; LUCENA, Carlos José Pereira de. Modelo 3C de Colaboração para o desenvolvimento de Sistemas Colaborativos. III Simpósio Brasileiro de Sistemas Colaborativos. Natal, RN, pp. 58-67, novembro de 2006.

QUADROS, Ronice Müller de; SCHMIEDT, Magali L. P. Ideias para ensinar português para alunos surdos. Brasília : MEC, SEESP, 2006. 120p.

SOUZA, G. Ambientes de Engenharia de Software Orientados a Corporação. Tese de Doutorado, COPPE, UFRJ, Rio de Janeiro, Brasil, 2008. 